\title{
Apparent Non-Cited Overlap between Two Published Articles by the Same Group of Authors
}

Recently, a reader questioned the apparent non-cited overlap between 2 published articles by the same group of authors:

- Tanriverdi H, et al.: Effect of homocysteine-induced oxidative stress on endothelial function in coronary slow-flow. Cardiology 2007;107:313-320, and

- Evrengul H, et al.: Interaction of plasma homocysteine and thyroid hormone concentrations in the pathogenesis of the slow coronary flow phenomenon. Cardiology 2007;108:186-192.

We queried the authors, specifically asking:

- Do the papers derive from the same study, protocol, and patient populations?

- If these are from the same study and patients, why was this not mentioned in the later paper and why was the earlier paper not cited?

- If these are from the same study and patients, why was the additional information and related methodology about thyroid hormones not included in the earlier paper?

We received the response paraphrased below:

These 2 articles included different data from the same study population and were prepared and submitted in very close proximity to each other.
Because review and decision had not been completed for the first article before the second manuscript was submitted, we could not provide a reference for the first paper in the second. (Editors' note: it might have been appropriate to notify the journal so that the first paper could have been cited in the second, once the first paper had been accepted, but this was not done.)

Though the study population was the same for both papers, we studied the effect of homocysteine-induced oxidative stress on endothelial function in coronary slowflow in the first paper, a subject that we continue to believe is not sufficiently related to the effect of thyroid hormone on slow coronary flow, reported in the second paper, to justify their inclusion in a single report. We believe it is common to report unrelated results of interest from single populations.

The Editors believe that it would have been preferable for the authors to have notified us about the need to add a reference and some explanatory information to the second paper before publication once the first paper was known to be accepted. However, we believe this error is not sufficient to warrant censure or any other action by Cardiology in this case.

The Editors

\begin{tabular}{ll}
\hline KARGER & ( ) 2010 S. Karger AG, Basel \\
Fax $+41608-6312 / 10 / 1173-0197 \$ 26.00 / 0$ \\
$\begin{array}{l}\text { E-Mail karger@karger.ch } \\
\text { www.karger.com }\end{array}$ & $\begin{array}{l}\text { Accessible online at: } \\
\text { www.karger.com/crd }\end{array}$
\end{tabular}

